\title{
Efectos genotóxicos de las resinas en odontología: Revisión de literatura
}

\section{Genotoxic effect of composites resins in dentistry: A review}

\author{
Hernández González D*, Méndez Silva J**, Díaz Caballero A***
}

\section{RESUMEN}

Objetivo: Analizar la evidencia científica disponible acerca de la genotoxicidad causada por los monómeros dentales liberados de las resinas compuestas.

Materiales y métodos: Se lleva a cabo una búsqueda electrónica exhaustiva de estudios relacionados con la genotoxicidad de las resinas compuestas o los monómeros dentales utilizando las bases de datos Pubmed, OvidSP, EbscoHost, SciVerse y SpringerLink. Se revisaron un total de 115 artículos y se seleccionaron 36 entre estudios clínicos controlados, estudios en animales y pruebas in vitro.

Conclusiones: La exposición a los monómeros residuales liberados por las resinas compuestas, causa gran preocupación por los estudios que advierten su potencial genotóxico. El principal mecanismo es atribuido al estrés oxidativo que se genera por aumento de las concentraciones de radicales libres y disminución de los niveles de glutatión, agente responsable del balance redox en la célula. Estas alteraciones conllevan a la activación de varias vías de apoptosis celular y retrasos en las fases G1 y G2 del ciclo celular.

Palabras clave: Genotoxicidad, micronúcleos con defecto cromosómico, metacrilatos, resinas compuestas, ensayo cometa (DeCS).

Palabras clave: Genotoxicidad, micronúcleos con defecto cromosómico, metacrilatos, resinas compuestas, ensayo cometa (DeCS).

\section{SUMMARY}

Objective: To examine the available scientific evidence about the genotoxicity caused by monomers released from dental composite resins.

Materials and methods: We carried out a comprehensive electronic search of studies related to the genotoxicity of composite resins or dental monomers using the databases PubMed, OvidSP, EBSCOhost, and SciVerseSpringerLink. We reviewed a total of 115 articles and 36 were selected from controlled clinical studies, animal studies and in vitro tests.

Conclusions: Exposure to residual monomers released from composites, causes great concern about studies that warn their genotoxic potential. The main mechanism is attributed to oxidative stress is generated by increasing concentrations of free radicals and decreased levels of Glutathione redox agent responsible for the cell balance. These changes lead to the activation of multiple pathways of apoptosis and delays in G1 and G2 phases of the cell cycle.

Key words: Genotoxicity tests, micronucleus test, comet assay, bisphenol A-glycidyl methacrylate, composites resins $(\mathrm{MeSH})$.

* Odontólogo. Facultad de Odontología. Universidad de Cartagena. Joven Investigador e innovador año 2013, Colciencias.

** Rehabilitador Oral, Docente Facultad de Odontología, Universidad de Cartagena.

*** Candidato a doctor en Ciencias Biomédicas. Docente Facultad de Odontología. Universidad de Cartagena. 
Grupo de investigación en Materiales Dentales de la Facultad de Odontología de la Universidad de Cartagena - MADEFOUC.

Grupo interdisciplinario de investigaciones y tratamientos odontológicos de la Universidad de Cartagena - GITOUC.

Fecha de recepción: 15 de abril de 2013.

Aceptado para publicación: 21 de mayo de 2013.

Hernández González D, Méndez Silva J, Díaz Caballero A. Efectos genotóxicos de las resinas en odontología: Revisión de literatura. Av. Odontoestomatol 2014; 30 (1): 29-38.

\section{INTRODUCCIÓN}

Con el desarrollo de la adhesión a los tejidos dentales, las resinas compuestas revolucionaron la odontología desde hace aproximadamente 50 años (1). Esto coincide con la preocupación de los dentistas a nivel mundial sobre la biocompatibilidad de la amalgama de plata, debido que, los vapores de mercurio liberado por ésta, causan alteraciones en los sistemas nervioso, renal o respiratorio (2). Por lo tanto, unos de los motivos principales para el cambio de este tipo de obturador dental por resinas compuestas de fotocurado era la seguridad biológica (3), sin apartar los requerimientos estéticos que cada vez tenían mayor auge conforme se desarrollaban mejores propiedades ópticas para estos materiales resinosos (1). Sin embargo, la biocompatibilidad a nivel genético de las resinas compuestas no es clara ya que estudios afirman que la acción genotóxica de éstas es causada por liberación de monómeros residuales al medio oral, los cuales surgen de la polimerización incompleta del material $(4,5)$ causando una serie de alteraciones metabólicas a nivel cromosomal comparables con las que pueden presentar la amalgama de plata (6). Consecuentemente, la creciente prevalencia de cáncer en la cavidad oral (7), genera dudas sobre la inocuidad de varios materiales dentales de amplio uso en la población. En la mayoría de los casos, la etiología de esta patología es desconocida, por lo tanto, es necesario un monitoreo del daño cromosomal en células del epitelio bucal en contacto con materiales restaurativos como las resinas compuestas para determinar si pueden dar origen a esta u otras enfermedades (8).

Dichas sustancias elevan el riesgo de aparición de carcinomas y modificaciones en los tejidos cercanos o que se encuentran en contacto con ellas, así como también ciertos tejidos blanco distantes al lugar donde se está generando el estímulo carcinogénico, por lo tanto, es de enorme importancia determinar la relación riesgo-beneficio de las actuales resinas compuestas, ya que hasta el momento los beneficios en cuanto a la estética son palpables en la práctica clínica y en la satisfacción de los pacientes, sin embargo, el riesgo biológico aún no se esclarece de una manera satisfactoria, ya que son muy pocos los estudios que responden todas las dudas que generan estos materiales en cuanto al daño genético que pueden causar $(8,9)$. Es así como la presente revisión contribuye a complementar el conocimiento científico del cual podrían apoyarse posteriores investigaciones afines que divulguen la biocompatibilidad de las resina compuestas, teniendo en cuenta que, el rápido desarrollo actual de las tecnologías hace que se necesiten frecuentes evaluaciones y monitoreo de todos los cambios sustanciales en la composición de estos productos.

El objetivo de la presente revisión es analizar la evidencia científica disponible acerca de la genotoxicidad causada por los monómeros dentales liberados de las resinas compuestas, teniendo en cuenta su mecanismo de acción y las posibles vías por las cuales estos agentes afectan al organismo. Además se analizan las distintas pruebas mediante las cuales podemos evaluar este fenómeno.

\section{MATERIALES Y MÉTODOS}

Se realizó una búsqueda electrónica de estudios relacionados con la genotoxicidad de las resinas compuestas o de los monómeros dentales, del año 
2000 hasta el 2012. Las bases de datos utilizadas fueron Pubmed, OvidSP, EbscoHost, SciVerse y SpringerLink. Se usó el operador booleano "AND" entre las siguientes palabras clave $\mathrm{MeSH}$ : Genotoxicity tests, micronucleus test, comet assay, bisphenol AGlycidyl methacrylate, composites resins.

Se revisaron un total de 115 artículos y se seleccionaron 36 entre estudios clínicos controlados, estudios en animales y pruebas in vitro.

\section{RESULTADOS}

\section{Elusión de los monómeros a la cavidad oral}

Polydorou et al. determinaron mediante pruebas in vitro la elusión de monómeros en dos resinas compuestas de uso comercial (4). Encontrando que cerca del $50 \%$ de la cantidad total de TEGDMA liberado lo hacía en las primeras 24 horas y hasta 28 días después de su polimerización. Este mismo autor en un estudio posterior comparó in vitro varios materiales resinosos y concluyó que la cantidad de monómeros liberados disminuye después de 28 días y puede presentarse hasta después de 1 año de almacenamiento (5).

Michelsen et al. concluye mediante su estudio in vivo que la cantidad de monómero liberado en la saliva sólo se presenta las primeras 10 minutos después de su colocación. Este autor no encontró monómeros liberados después de 24 horas ni de 7 días, por lo tanto esto puede explicar la baja incidencia de efectos locales adversos relacionados con las resinas compuestas (10).

\section{Ensayo de micronúcleos}

Durante la división celular el material genético (ADN) contenido en el núcleo celular, se replica y divide equitativamente dando lugar a dos células hijas idénticas; este proceso puede producirse de manera deficiente debido a errores durante la replicación y posterior división del ADN, a roturas cromosómicas y al efecto de la radiación y de sustancias genotóxicas, produciéndose pérdida cromosómica y haciendo que el reparto del material genético no sea equitativo. Cuando esto ocurre, el material genético que se desprende y que, por tanto, queda excluido y no se incorpora correctamente al núcleo de la célula hija, origina un nuevo núcleo de menor tamaño que el primario denominado "micronúcleo" (MN), visible fácilmente al microscopio óptico. El material genético desprendido puede derivar de cromosomas enteros o, más frecuentemente, de fragmentos cromosómicos a céntricos que quedan excluidos de los núcleos de las nuevas células durante anafase mitótica.

La mucosa bucal provee una barrera contra los potenciales carcinógenos que son metabolizados en potenciales productos reactivos. Cerca del $90 \%$ de los cánceres son de origen epitelial, la mucosa bucal puede ser usada para monitorear eventos genotóxicos tempranos como resultado de carcinógenos potenciales que entran al cuerpo por ingestión o inhalación (11). Células bucales exfoliadas se usaron exitosamente mostrando los efectos genotóxicos del tabaco, tratamientos médicos como radioterapia, exposición ocupacional a potenciales mutagénicos y/o químicos carcinogénicos y para estudios de quimioprevención del cáncer (12).

\section{Ensayo cometa}

En general, el principio básico del ensayo es la migración del ADN en una matriz de agarosa bajo condiciones de electroforesis. Luego, al ser observada la célula al microscopio (por fluorescencia o por tinción con plata del material nuclear), presenta la apariencia de un cometa, con una cabeza (región nuclear) y cola (formada por fragmentos nucleares que han migrado en dirección del ánodo) por lo que este ensayo es también conocido como ensayo Cometa, debido al patrón de migración del ADN que se produce en las células dañadas. La detección de la migración del ADN alterado depende de varios parámetros, tales como: la concentración de la matriz de agarosa, el pH, la temperatura y duración del desenrollamiento, voltaje, amperaje y duración de la electroforesis (13). El protocolo de este ensayo ha quedado bien establecido para detectar daño al ADN, específicamente aquellos producidos por las rupturas de cadena, la formación de sitios lábiles al álcali, los entrecruzamientos ADN-ADN y ADN-proteínas y 
más recientemente para la evaluación de los mecanismos de reparación de daño oxidativo en el ADN en células eucariotas obtenidas tanto de estudios in vivo como in vitro.

Ostling y Johanson, en el año 1984, fueron los primeros en desarrollar una técnica de electroforesis en microgeles para detectar el daño al ADN a nivel de células individuales. En esta técnica las células son embebidas en agarosa y son añadidas en láminas de microscopio para ser sometidas a una electroforesis neutral, tras la lisis en presencia de sales y detergentes. Las células con una elevada frecuencia de rupturas de doble cadena (RDC) mostraron una significativa migración del ADN hacia el ánodo (13).

Las condiciones neutrales limitaron grandemente la utilidad del ensayo es por esto que, en 1988, Singh y cols. introdujeron una técnica en microgeles que involucra la electroforesis en condición alcalina $(\mathrm{pH}$ 13) para detectar el daño al ADN en células individuales (14). En estas condiciones de $\mathrm{pH}$ la incrementada migración del ADN es asociada con elevados niveles de rupturas de simples cadenas (RSC) asociadas con sitios de reparación por escisión incompletos y sitios lábiles al álcali (SLA). Como la mayoría de los agentes genotóxicos inducen en mayor magnitud RSC y/o SLA que RDC, esta versión del ensayo tiene mayor importancia en la identificación de los agentes genotóxicos. Dos años más tarde Olive, en el año 1990, introdujo otra versión alcalina de este ensayo, en la cual la electroforesis del

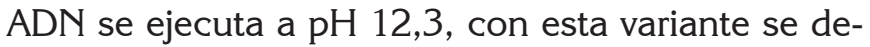
tectan SLA convertidos en RSC (15).

\section{Resinas compuestas}

Las resinas compuestas son usadas para una variedad de aplicaciones en odontología, básicamente están conformadas por una matriz polimérica, que típicamente es un metacrilato, un relleno de refuerzo hecho de vidrio radio opaco, un agente de unión Silano que une la matriz con el relleno y promotores o moduladores de polimerización. El monómero predominante usado en las resinas compuestas comerciales es el bis-GMA el cual posee una alta viscosidad, por lo tanto es mezclado con otros monómeros como TEGDMA y UDMA (1).

\section{Monómeros dentales}

Los monómeros pueden ser considerados el constituyente clave en la resina dental, su grado de conversión es un determinante importante de las fuerza físico-mecánica del polímero resultante. La conversión generalmente no es completa y se acepta que sea baja en las resinas compuestas y en los adhesivos. Aparte de menores fuerzas mecánicas, un bajo grado de conversión resulta en una alta permeabilidad, mayor absorción de agua, mayor microfiltración, mayor liberación de monómeros residuales y, por lo tanto, menor biocompatibilidad (16). La polimerización es inhibida por varios factores, tales como la presencia de oxígeno (resultando en la capa inhibida), la presencia de agua proveniente de la dentina, entre otros (16).

\section{Inducción de genotoxicidad causada por los monómeros dentales}

El ADN es el blanco molecular de los componentes de los materiales dentales resinosos, ya que sus efectos genotóxicos han sido detectados en bacterias y células de animales mostrando la interacción entre el ADN y los monómeros residuales o metabolitos asociados. A la fecha parece que hay una clara diferencia en el potencial genotóxico de varios metacrilatos, por ejemplo, no se encontró inducción de mutación genética causada por el Bis-GMA y el UDMA (17). Sin embargo, no se descarta que exista el daño a cierto nivel, ya que el Bis-GMA presentó resultados positivos al test de inhibición de síntesis de ADN realizado por Heil (18). Aunque, no se detectaron mutaciones genéticas con los monómeros HEMA y MMA, pero altas concentraciones de HEMA provocó mayores niveles de formación de micronúcleo indicando aberraciones cromosómicas in vitro en los estudios de Schweikl $(17,19)$, por lo tanto es probable que el HEMA también produzca daño al ADN. El monómero bifuncional TEGDMA causa efectos mutagénicos dosis-dependiente en cultivo de células de animales, además la frecuencia de mutación genética aumentó más de 10 veces y este monómero también indujo la formación de micronúcleo $(17,19)$, también la fractura de las cadenas de ADN por el TEGDMA y el HEMA fue indicado por la prueba cometa en los estudios de Kleinsasser et al. (20, 21). 
Los mecanismos moleculares que conducen a mutaciones por monómeros de resina no son claros en la actualidad. Sin embargo hay dos posibilidades para la generación de lesiones de ADN (Figura 1).

Primero, el espectro de mutación inducido por el TEGDMA es similar al causado en el genoma de las células animales después de la exposición a rayos $\mathrm{X}$ $\mathrm{y}$ varios agentes químicos, incluyendo las drogas anticancerígenas $(22,23)$. Segundo, TEGDMA y los monómeros relativos pueden inducir mutaciones por un mecanismo secundario vía generación de Especies de Óxido Reactivos (EOR) como lo hacen ciertos agentes tales como radiación ionizante, UV y ciertos químicos (24).

\section{Daño al ADN y regulación del ciclo celular}

El ADN mitocondrial y genómico son el blanco principal para agentes como EOR, luz ultravioleta, radiación iónica y sustancias químico-terapéuticas. Estos agentes genotóxicos causan una serie de lesiones que incluyen la rotura de cadena simple o doble de ADN. Como respuesta, un elaborado sistema de regulación molecular es activado para mantener la integridad genómica celular (25). El éxito de las fases G1, S y G2 en el ciclo de las células eucariotas es precisamente monitoreado por varios puntos de control. Estos activan respuestas celulares sobre el daño al ADN a través de ciertas proteínas $(26,27)$. Luego,

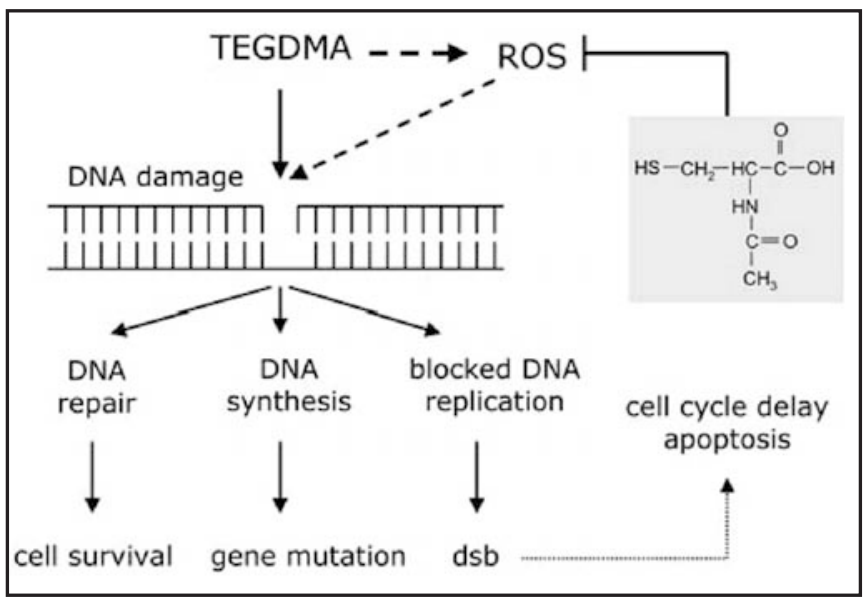

Fig. 1. Schweikl H et al. Genetic and cellular toxicology of dental resin monomers. Journal of Dental Research 2006;85(10):870-7. el ciclo celular puede ser bloqueado para reparar el ADN dañado o para conducir a la célula a una muerte programada. Sin embargo, los defectos en la señalización de los puntos de control del ciclo celular pueden ser desastrosos para la integridad del genoma, debido a que pueden conducir a un daño genético irreversible como mutaciones o daño a los cromosomas. La regulación de los puntos de control del ciclo celular es una red con mecanismos complejos realizados por vías dependientes y no dependientes de P53 (26).

El comonómero TEGDMA ha sido mostrado por Schweikl et al. como el causante de acumulación reversible de células de la línea V79 en fase G2, probablemente debido a la falta de un punto de control funcional en la fase G1 en esta línea celular, por lo tanto, una fase G1 prolongada causada por altas concentraciones de TEGDMA es probablemente estabilizada por un punto de control dependiente de P53 (28).

En un estudio de Wisniewska evaluó la acción conjunta del TEGDMA y UDMA sobre células de hámster, encontrando que estos monómeros causan por separado una inducción de daños citotóxicos y genotóxicos, sin embargo su asociación no incrementa su acción dañina (29).

\section{DISCUSIÓN}

Inicialmente mediante estudios in vitro varios autores evaluaron la capacidad citotóxica y genotóxica de los monómeros dentales, aunque sus mecanismos no están totalmente claros, recientes hallazgos identifican al dimetacrilato de trietilenglicol comonómero (TEGDMA) y el 2 hidroxietil metacrilato (HEMA), entre otros, como causantes de alteraciones en el ciclo celular mediante la inhibición de los niveles de glutatión (GSH), el cual funciona como antioxidante natural protegiendo la célula de los daños causado por especies reactivas de oxígeno (ROS), quienes pueden activar diferentes vías de apoptosis celular (8, 21, 30-35). La transformación del daño en el ADN a mutación no suele ser inmediata sino a largo plazo (32) y, en determinadas concentraciones estos monómeros dentales tienen un alto potencial carcinogénico $(33,35,36)$. 
Schweikl et al. encontraron en varios estudios in vitro, un aumento del número de formación de micronúcleos en cultivos de células de fibroblastos en contacto con diferentes concentraciones de BisGMA, TEGDMA y HEMA $(19,28,36)$. Drozdz et al., mediante un estudio in vitro en donde evalúa la acción del Bis-GMA en linfocitos humanos, encontró que este puede llegar a causar un amplio espectro de daño al ADN mediante la interacción directa con este causando una rotura de cadena doble, por lo tanto se puede concluir que esto causa el retraso en la fase $S$ del ciclo celular (37). De esta misma manera Szczepanska et al. entró daño genotóxico causado por estos compuestos en fibroblastos gingivales (38).

Investigaciones en animales como los de Arossi et al. corroboraron la actividad mutagénica de estos compuestos, utilizando el test de recombinación y mutación somática (S.M.A.R.T) en Drosofila Melanogaster ya que su genética es homóloga a la de los mamíferos $(39,40)$. Varios estudios notificaron conclusiones similares en cuanto a la medición en mamíferos del daño al material genético causado por los monómeros de uso odontológico (41).

Posteriormente, pruebas en humanos como la de Di Pietro et al. buscaron evaluar la actividad genotóxica de estos materiales restauradores mediante ensayo cometa en sangre periférica de personas jóvenes, comparando los resultado obtenidos con un grupo control. Encontraron que la amalgama y los metacrilatos tenían un potencial adverso sobre la salud humana mediante el mecanismo de lesiones oxidativas al ADN, razón por la cual sugieren estudios clínicos que involucren otros indicadores específicos de daño oxidativo al ADN (6).

En conclusión podemos afirmar que el análisis de la toxicología celular de los monómeros dentales ha dado una nueva percepción en la interpretación de los factores de riesgo de los tejidos de la cavidad oral. Los esfuerzos hechos en el campo de la toxicología celular de los monómeros y los hallazgos futuros derivados de la biología celular pueden mejorar nuestro conocimiento de los efectos de estos compuestos sobre los tejidos humanos. Últimamente se ha hecho mucho énfasis en estudiar la relevancia clínica de identificar el potencial de inducir daño ce- lular y tisular provocado por los materiales dentales y sus componentes para conocer desde un punto de vista molecular la acción de los compuestos xenobióticos que son colocados en cavidad oral por el personal odontológico, cobra gran importancia al encontrar evidencia del daño a nivel genético que estos pueden causar. Sabiendo que desde el contexto ético, debemos ofrecer a nuestros pacientes tratamientos de alta calidad y con los menores efectos secundarios posibles.

\section{AGRADECIMIENTOS}

Agradecimiento al Departamento Administrativo de Ciencia, Tecnología e Innovación COLCIENCIAS, por su compromiso con el fomento de la investigación mediante el programa de Jóvenes Investigadores e Innovadores año 2012-2013.

\section{BIBLIOGRAFÍA}

1. Ferracane JL. Resin composite-state of the art. Dental materials: official publication of the Academy of Dental Materials 2011 Jan;27(1):2938.

2. Bernhoft RA. Mercury toxicity and treatment: a review of the literature. Journal of environmental and public health. 2012;2012:460-508.

3. Mutter J, Naumann J, Sadaghiani C, Walach H, Drasch G. Amalgam studies: disregarding basic principles of mercury toxicity. International journal of hygiene and environmental health 2004 Sep; 207(4):391-7.

4. Polydorou O, Trittler R, Hellwig E, Kummerer K. Elution of monomers from two conventional dental composite materials. Dental materials: official publication of the Academy of Dental Materials 2007 Dec;23(12):1535-41.

5. Polydorou O, Konig A, Hellwig E, Kummerer K. Long-term release of monomers from modern dental-composite materials. European journal of oral sciences 2009 Feb;117(1):68-75. 
Hernández González D, Méndez Silva J, Díaz Caballero A.

6. Di Pietro A, Visalli G, La Maestra S, Micale R, Baluce B, Matarese G, et al. Biomonitoring of DNA damage in peripheral blood lymphocytes of subjects with dental restorative fillings. Mutation research 2008 Feb 29;650(2):115-22.

7. Wunsch-Filho V. The epidemiology of oral and pharynx cancer in Brazil. Oral oncology 2002 Dec;38(8):737-46.

8. Schweikl H, Spagnuolo G, Schmalz G. Genetic and cellular toxicology of dental resin monomers. Journal of dental research 2006 Oct;85(10):870-7.

9. Goldberg $M$. In vitro and in vivo studies on the toxicity of dental resin components: a review. Clinical oral investigations 2008 Mar;12(1):1-8.

10. Michelsen VB, Kopperud HB, Lygre GB, Bjorkman L, Jensen E, Kleven IS, et al. Detection and quantification of monomers in unstimulated whole saliva after treatment with resin-based composite fillings in vivo. European journal of oral sciences 2012 Feb;120(1):89-95.

11. Chatterjee S, Dhar S, Sengupta B, Ghosh A, De $M$, Roy S, et al. Cytogenetic monitoring in human oral cancers and other oral pathology: the micronucleus test in exfoliated buccal cells. Toxicology mechanisms and methods 2009 Sep;19(6-7):427-33. PubMed PMID: 19778244. eng.

12. Suhas S, Ganapathy KS, Gayatri Devi M, Ramesh C. Application of the micronucleus test to exfoliated epithelial cells from the oral cavity of beedi smokers, a high-risk group for oral cancer. Mutation research. 2004 Jul 11;561(1-2):15-21. PubMed PMID: 15238226. eng.

13. Tice RR, Agurell E, Anderson D, Burlinson B, Hartmann A, Kobayashi H, et al. Single cell gel/ comet assay: guidelines for in vitro and in vivo genetic toxicology testing. Environmental and molecular mutagenesis 2000;35(3):206-21. PubMed PMID: 10737956. eng.

14. Singh NP, McCoy MT, Tice RR, Schneider EL. A simple technique for quantitation of low levels of
DNA damage in individual cells. Experimental cell research 1988 Mar;175(1):184-91. PubMed PMID: 3345800. eng.

15. Olive P, Banath J, Durand R. Heterogeneity in radiation-induced DNAdamage and repair in tumor and normal cells using the "comet" assay. Radiat Res 1990 (122):86-94.

16. Van Landuyt K. Systematic review of the chemical composition of contemporary dental adhesives. Biomaterials 2007 Sep;28(26):3757-85. PubMed PMID: 17543382.

17. Schweikl H, Schmalz G, Rackebrandt K. The mutagenic activity of unpolymerized resin monomers in Salmonella typhimurium and V79 cells. Mutation research 1998 Jul 8;415(1-2):11930. PubMed PMID: 9711268. eng.

18. Heil J, Reifferscheid G, Waldmann P, Leyhausen G, Geurtsen W. Genotoxicity of dental materials. Mutation research 1996 Jul 5;368(3-4):181-94. PubMed PMID: 8692224. eng.

19. Schweikl H, Schmalz G, Spruss T. The induction of micronuclei in vitro by unpolymerized resin monomers. Journal of dental research 2001 Jul;80(7):1615-20. PubMed PMID: 11597020. eng.

20. Kleinsasser $\mathrm{NH}$, Schmid K, Sassen AW, Harreus UA, Staudenmaier R, Folwaczny M, et al. Cytotoxic and genotoxic effects of resin monomers in human salivary gland tissue and lymphocytes as assessed by the single cell microgel electrophoresis (Comet) assay. Biomaterials 2006 Mar;27(9):1762-70. PubMed PMID: 16242184. eng.

21. Kleinsasser $\mathrm{NH}$, Wallner $\mathrm{BC}$, Harreus UA, Kleinjung $T$, Folwaczny $M$, Hickel $R$, et al. Genotoxicity and cytotoxicity of dental materials in human lymphocytes as assessed by the single cell microgel electrophoresis (comet) assay. Journal of dentistry 2004 Mar;32(3):229-34. PubMed PMID: 15001288. eng.

22. Lavin MF, Birrell G, Chen P, Kozlov S, Scott S, Gueven N. ATM signaling and genomic stability 
in response to DNA damage. Mutation research 2005 Jan 6;569(1-2):123-32. PubMed PMID: 15603757. eng.

23. Pfeiffer P, Feldmann E, Odersky A, Kuhfittig-Kulle S, Goedecke W. Analysis of DNA double-strand break repair by nonhomologous end joining in cell-free extracts from mammalian cells. Methods Mol Biol 2005;291:351-71. PubMed PMID: 15502235. eng.

24. Achanta G, Huang P. Role of p53 in sensing oxidative DNA damage in response to reactive oxygen species-generating agents. Cancer research 2004 Sep 1;64(17):6233-9. PubMed PMID: 15342409. eng.

25. Shackelford RE, Kaufmann WK, Paules RS. Cell cycle control, checkpoint mechanisms, and genotoxic stress. Environmental health perspectives 1999 Feb;107 Suppl 1:5-24. PubMed PMID: 10229703. Pubmed Central PMCID: 1566366. eng.

26. Nyberg KA, Michelson RJ, Putnam CW, Weinert TA. Toward maintaining the genome: DNA damage and replication checkpoints. Annual review of genetics 2002;36:617-56. PubMed PMID: 12429704. eng.

27. Sancar A, Lindsey-Boltz LA, Unsal-Kacmaz K, Linn S. Molecular mechanisms of mammalian DNA repair and the DNA damage checkpoints. Annual review of biochemistry 2004;73:39-85. PubMed PMID: 15189136. eng.

28. Schweikl H, Altmannberger I, Hanser N, Hiller KA, Bolay C, Brockhoff G, et al. The effect of triethylene glycol dimethacrylate on the cell cycle of mammalian cells. Biomaterials 2005 Jul;26 (19):4111-8. PubMed PMID: 15664638. eng.

29. Wisniewska-Jarosinska M, Poplawski T, Chojnacki CJ, Pawlowska E, Krupa R, Szczepanska J, et al. Independent and combined cytotoxicity and genotoxicity of triethylene glycol dimethacrylate and urethane dimethacrylate. Molecular biology reports 2011 Oct;38(7):4603-11. PubMed PMID: 21127987.
30. Schweikl H, Hartmann A, Hiller KA, Spagnuolo G, Bolay C, Brockhoff G, et al. Inhibition of TEGDMA and HEMA-induced genotoxicity and cell cycle arrest by $\mathrm{N}$-acetylcysteine. Dental materials: official publication of the Academy of Dental Materials 2007 Jun;23(6):688-95. PubMed PMID: 16890983. eng.

31. Chang HH, Guo MK, Kasten FH, Chang MC, Huang GF, Wang YL, et al. Stimulation of glutathione depletion, ROS production and cell cycle arrest of dental pulp cells and gingival epithelial cells by HEMA. Biomaterials 2005 Mar; 26(7):745-53. PubMed PMID: 15350779. eng.

32. Poplawski T, Pawlowska E, Wisniewska-Jarosinska M, Ksiazek D, Wozniak K, Szczepanska J, et al. Cytotoxicity and genotoxicity of glycidyl methacrylate. Chemico-biological interactions 2009 Jun 15;180(1):69-78.

33. Pawlowska E, Poplawski T, Ksiazek D, Szczepanska J, Blasiak J. Genotoxicity and cytotoxicity of 2-hydroxyethyl methacrylate. Mutation research 2010 Feb;696(2):122-9. PubMed PMID: 20079459. eng.

34. Kaya A, Undeger U, Aydin S, Omurlu H, Basaran N. Genotoxicity evaluation of dentine bonding agents by comet assay. International endodontic journal 2011 Sep;44(9):807-16. PubMed PMID: 21477155. eng.

35. Poplawski T, Loba K, Pawlowska E, Szczepanska J, Blasiak J. Genotoxicity of urethane dimethacrylate, a tooth restoration component. Toxicology in vitro: an international journal published in association with BIBRA 2010 Apr;24(3):854-62. PubMed PMID: 20005290. eng.

36. Schweikl H, Hiller KA, Bolay C, Kreissl M, Kreismann W, Nusser A, et al. Cytotoxic and mutagenic effects of dental composite materials. Biomaterials 2005 May;26(14):1713-9. PubMed PMID: 15576145. eng.

37. Drozdz K, Wysokinski D, Krupa R, Wozniak K. Bisphenol A-glycidyl methacrylate induces a 
broad spectrum of DNA damage in human lymphocytes. Archives of toxicology 2011 Nov;85(11):1453-61.

38. Szczepanska J, Poplawski T, Synowiec E, Pawlowska E, Chojnacki CJ, Chojnacki J, et al. 2-hydroxylethyl methacrylate (HEMA), a tooth restoration component, exerts its genotoxic effects in human gingival fibroblasts trough methacrylic acid, an immediate product of its degradation. Molecular biology reports 2012 Feb; 39(2):1561-74.

39. Arossi GA, Lehmann M, Dihl RR, Reguly ML, de Andrade $\mathrm{HH}$. Induced DNA damage by dental resin monomers in somatic cells. Basic $\mathcal{E}$ clinical pharmacology $\varepsilon$ toxicology 2010 Feb;106(2): 124-9. PubMed PMID: 20041880. eng.

40. Arossi GA, Dihl RR, Lehmann M, Cunha KS, Reguly ML, de Andrade HH. In vivo genotoxicity of dental bonding agents. Mutagenesis 2009
Mar;24(2):169-72. PubMed PMID: 19047495. eng.

41. Scarparo RK, Grecca FS, Fachin EV. Analysis of tissue reactions to methacrylate resin-based, epoxy resin-based, and zinc oxide-eugenol endodontic sealers. Journal of endodontics 2009 Feb;35(2): 229-32. PubMed PMID: 19166779. eng.

\section{CORRESPONDENCIA}

Daniel Fernando Hernández González

Facultad de Odontología. Universidad de Cartagena. Sede Zaragocilla.

Campus de la Salud.

Avenida El Consulado Calle 30, No. 48-152

Cartagena de Indias DT y C-Colombia

Correos electrónicos: danielhdez9@gmail.com; todi63@gmail.com 